\title{
A Blood-based Gene-expression Scoring System for Cancer Screening in Patients With Branch-duct Intraductal Papillary Mucinous Neoplasms
}

\author{
REI SUZUKI ${ }^{1}$, HIROSUMI TAMURA $^{2}$, REIKO HONMA ${ }^{3}$, NAOKI KONNO ${ }^{1}$, HIROKI IRIE $^{1}$, \\ TADAYUKI TAKAGI ${ }^{1}$, MITSURU SUGIMOTO ${ }^{1}$, HIROYUKI ASAMA ${ }^{1}$, YUKI SATO ${ }^{1}$, \\ OKUBO YOSHINORI ${ }^{4}$, JUN NAKAMURA ${ }^{4}$, MIKA TAKASUMI ${ }^{1}$, TSUNETAKA KATO ${ }^{4}$, \\ MINAMI HASHIMOTO ${ }^{4}$, TAKUTO HIKICHI ${ }^{4}$, JUN-ICHI IMAI ${ }^{2}$, SHINYA WATANABE ${ }^{2}$ and HIROMASA OHIRA ${ }^{1}$ \\ ${ }^{1}$ Department of Gastroenterology, Fukushima Medical University School of Medicine, Fukushima, Japan; \\ ${ }^{2}$ Translational Research Center, Fukushima Medical University, Fukushima, Japan; \\ ${ }^{3}$ Nippon Gene Co., Ltd., Tokyo, Japan; \\ ${ }^{4}$ Department of Endoscopy, Fukushima Medical University Hospital, Fukushima, Japan
}

\begin{abstract}
Background: In patients with branch-duct intraductal papillary mucinous neoplasms (BD-IPMN), we aimed to develop a novel blood-based biomarker utilizing a gene-expression profile for the detection of pancreatic malignancies, such as IPMN-derived carcinoma (IPMC) or pancreatic ductal adenocarcinoma (PDAC). Patients and Methods: We enrolled 40 patients with pancreatic tumors (24 $B D-I P M N s$, four IPMCs and 12 PDACs) and identified the characteristic gene-expression profiles in pancreatic malignancies. Subsequently, we constructed a gene-expression scoring system for the proper diagnosis of pancreatic malignancies. The result was validated in 14 patients (five IPMNs, three IPMCs and six PDACs). Results: The scoring system utilizing the expression levels of 13 genes showed high diagnostic yield (sensitivity $=94.0 \%$, specificity $=92.0 \%$ and area under the curve=0.94), which was confirmed in the validation set. Furthermore, its diagnostic yield was not reduced even in early-stage pancreatic malignancies (sensitivity $=85.0 \%$, specificity $=93.0 \%$ and area under the curve $=0.88$ ). Conclusion: We developed a blood-based gene expression scoring system for cancer screening in patients with BD-IPMNs.
\end{abstract}

This article is freely accessible online.

Correspondence to: Rei Suzuki, MD, Ph.D., Assistant Professor, Department of Gastroenterology, Fukushima Medical University School of Medicine, 1 Hikarigaoka, Fukushima 960-1295, Japan. Tel: +81 245471202, Fax: +81 245472055, e-mail: subaru@fmu.ac.jp

Key Words: Intraductal papillary mucinous neoplasms, IPMNderived carcinoma, pancreatic ductal adenocarcinoma, biomarker, microarray.
Intraductal papillary mucinous neoplasms (IPMNs) can be classified into two types based on their primary location in the pancreatic duct: Main-duct IPMN and branch-duct (BD-IPMN) (1). Each type differs in the risk of malignancy, and the revised 2017 international consensus guideline recommended resection for all surgically fit patients with main duct-IPMN since it has a high risk of malignancy (2). On the other hand, BD-IPMN has a lower frequency of malignancy, and careful follow-up with imaging modalities is generally recommended. However, recent studies reported a surprisingly high cumulative risk of pancreatic malignancies such as concomitant PDAC and IPMNderived carcinoma (IPMC) during the observational period in patients with BD-IPMN. A recent long-term observational study reported that the overall incidence rates of pancreatic malignancies 5, 10, and 15 years after BD-IPMN diagnosis were up to $3.3 \%, 6.6 \%$ and $15.0 \%$, respectively (3). Therefore, a careful surveillance program that aims to detect pancreatic malignancies should be established for patients with BD-IPMN.

Several guidelines have been developed and are widely used to estimate the malignant potential of BD-IPMN mainly for the detection of IPMC $(2,4,5)$. The guidelines have proposed several key imaging features (e.g. mural nodule or dilated pancreatic duct) obtained by high-resolution imaging modalities for risk stratification. However, interpreting the results of these sophisticated modalities (e.g. endoscopic ultrasound or magnetic resonance imaging) requires expertise, and the diagnostic performance of the guidelines was reported to be varied in distinguishing benign IPMN and IPMC (sensitivity range from 7.3 to $83.3 \%$ and specificity range from 35.2 to $88.2 \%$ ) (6-10). Regarding concomitant PDAC, little evidence exists for a screening program.

Highly diagnostic biomarkers that can be obtained in a less invasive manner are an ideal method for cancer screening 
Table I. Clinical background of the patients.

\begin{tabular}{|c|c|c|c|c|c|c|}
\hline & \multicolumn{3}{|c|}{ Discovery set } & \multicolumn{3}{|c|}{ Validation set } \\
\hline & IPMN (n=24) & IPMC+PDAC $(n=16)$ & $p$-Value & IPMN $(\mathrm{n}=5)$ & IPMC+PDAC $(n=9)$ & $p$-Value \\
\hline Age, years & $72.5 \pm 10.2$ & $74.4 \pm 2.2$ & 0.49 & $71.0 \pm 14.4$ & $72.4 \pm 7.3$ & 0.80 \\
\hline Make, n (\%) & $14(58.3)$ & $12(75.0)$ & 0.33 & $14(58.3)$ & $12(75.0)$ & 0.57 \\
\hline Location - Ph, n (\%) & $10(41.7)$ & $10(62.5)$ & 0.33 & $10(41.7)$ & $10(62.5)$ & 0.87 \\
\hline Height, cm & $159.8 \pm 11.1$ & $161.7 \pm 2.2$ & 0.58 & $155.9 \pm 6.2$ & $157.2 \pm 10.1$ & 0.76 \\
\hline Weight, kg & $59.5 \pm 14.4$ & $52.7 \pm 2.4$ & 0.10 & $51.4 \pm 6.9$ & $54.8 \pm 9.6$ & 0.50 \\
\hline Current smoker, n (\%) & $5(20.8)$ & $5(31.3)$ & 0.48 & $0(0)$ & $2(22.2)$ & 0.25 \\
\hline Family Hx of PDAC, n (\%) & $3(12.5)$ & $0(0)$ & 0.26 & $0(0)$ & $1(11.1)$ & 0.43 \\
\hline $\mathrm{CEA}, \mathrm{ng} / \mathrm{ml}$ & $3.2 \pm 0.4$ & $78.9 \pm 43.5$ & 0.04 & $2.3 \pm 0.8$ & $28.1 \pm 51.5$ & 0.17 \\
\hline CA19-9, U/ml & $15.2 \pm 6.3$ & $19,706.0 \pm 13,633.0$ & 0.001 & $9.7 \pm 14.4$ & $17,136.7 \pm 43,704.0$ & 0.43 \\
\hline cStage I-II*, n (\%) & NA & $4(25.0)$ & & NA & $3(33.3)$ & \\
\hline
\end{tabular}

IPMN: Intraductal papillary mucinous neoplasm; IPMC: IPMN-derived carcinoma; PDAC: pancreatic ductal adenocarcinoma; M: male; Ph: head of the pancreas; Hx: history; CEA: carcinoembryonic antigen; CA19-9: carbohydrate antigen 19-9; NA not applicable. *American Joint Committee on Cancer/Union for International Cancer Control, eighth edition $(19,20)$. Statistically significant $p$-values are shown in bold.

programs. A considerable number of studies have attempted to develop biomarkers of body fluids (blood, pancreatic juice and cystic fluid) for the risk stratification of IPMN (11-16). A recent systematic review, including 193 studies with 12,297 patients, reported that serum carcinoembryonic antigen (CEA) and carbohydrate antigen 19-9 (CA19-9) had moderate diagnostic accuracy among other biomarkers (of 0.80 and 0.81, respectively) (17). Those serum biomarkers can be obtained less invasively but should be complementary to other imaging modalities for IPMN, considering their low diagnostic yield.

We previously developed a novel gene-expression scoring system that helped in differentiating basaloid squamous cell carcinoma of the esophagus from non-basaloid squamous cell carcinoma with high accuracy (18). In this study, we applied this gene-scoring system using blood specimens for detection of both IPMC and PDAC in patients with BD-IPMN.

\section{Patients and Methods}

Patients and diagnosis. To establish a novel gene-expression scoring system for the accurate diagnosis of pancreatic malignancies in patients with BD-IPMN, we prospectively enrolled 86 patients who were diagnosed with pancreatic tumors (32 BD-IPMN, 11 IPMC, 40 PDAC and three other types) by imaging modalities at Fukushima Medical University Hospital between April 2017 and April 2019. Among them, we excluded patients who met the following criteria: (i) Current treatment for malignancies; (ii) presence of concomitant non-pancreatic malignancy; and (iii) age younger than 18 years. Finally, we enrolled 40 patients (24 BDIPMNs, four IPMCs and 12 PDACs) for the initial study (discovery set). Next, we enrolled 14 consecutive patients diagnosed with pancreatic tumors (five IPMNs, three IPMCs and six PDACs) between April 2019 and December 2019 to validate the results of the scoring system under the same criteria (validation set). The clinical backgrounds of the patients in the training and validation sets are summarized in Table I. In brief, there were significant differences in most variables, and the serum levels of CEA and CA19-9 were lower in patients with IPMN than in patients with pancreatic malignancies in both sets.

The primary endpoint was diagnostic accuracy, which differentiated IPMN and pancreatic malignancies with the gene-expression scoring system. The secondary endpoints were sensitivity and specificity for the gene-expression scoring system and other tumor markers.

The diagnostic criteria of BD-IPMN were as follows: (i) Cystic dilation of the branch duct connected to the main pancreatic duct with/without (ii) secretion of mucin from the major or minor papilla identified by endoscopic retrograde cholangiopancreatography (ERCP) or duodenoscopy (12). Regarding IPMC, the diagnosis was made by histopathology of surgical specimens or clinical features highly suggestive of the malignant transformation of BD-IPMN (progression of BD-IPMN with intramural nodules and invasion to surrounding organs). All PDAC cases were pathologically diagnosed via endoscopic ultrasound-guided fine-needle aspiration. The clinical stage (cStage) of pancreatic malignancies was determined according to the eighth edition of the American Joint Committee on Cancer (AJCC)/Union for International Cancer Control (UICC) staging system $(19,20)$.

Patient clinical data, including age, sex, location of disease, height, body weight, smoking and drinking habits, family history of pancreatic cancer and serum levels of CEA and CA19-9, were obtained from the electronic medical records. The pathological characteristics of the tumors (e.g. cStage) were also retrieved.

The study protocol conformed to the ethical guidelines of the 1975 Declaration of Helsinki and was approved by the Ethics Committee of Fukushima Medical University (approval no. 1953). Written informed consent was obtained from the patients.

RNA preparation. Aspiration of peripheral blood was conducted before treatment for pancreatic tumors in all cases. Peripheral blood was obtained through a venous catheter. A sample of peripheral blood of 1 to $2 \mathrm{ml}$ was immediately mixed vigorously with three volumes of ISOGEN-LS (Nippon Gene Co., Ltd., Tokyo, Japan). After adding $11 \mathrm{ml}$ of ISOGEN (Nippon Gene) to each sample, the 
samples were mixed thoroughly, and total RNA extraction was conducted according to the manufacturer's protocol (18).

Comprehensive gene-expression analysis (CGEA). For the DNA microarray that used total RNA, a set of synthetic polynucleotides (80-mers) (MiroDiagnostic, Tokyo, Japan) representing 14,400 species of human transcript sequences was printed on a glass slide using a custom arrayer. For the RNA of the samples, SuperScript II (Invitrogen Life Technologies, Carlsbad, CA, USA) and cyanine 5dUTP (Perkin-Elmer Inc., Boston, MA, USA) were used to synthesize labeled cDNA from $5 \mu \mathrm{g}$ of total RNA. Using the same method for the reference RNA, cyanine 3-dUTP (Perkin-Elmer Inc.) was used to synthesize labeled cDNA from $5 \mu \mathrm{g}$ of Human Universal Reference RNA Type II (MicroDiagonostic).

Hybridization was performed with a labeling and hybridization kit (MicroDiagnostic). Signals were measured using a GenePix 4000B Scanner (Axon Instruments, Inc., Union City, CA, USA) and then processed into the primary expression ratios of the cyanine 5 intensity of each specimen to the cyanine 3 intensity of the human common reference RNA. Each ratio was normalized using GenePix Pro 3.0 software (Axon Instruments, Inc.). The primary expression ratios were converted into $\log _{2}$ values, which were designated as $\log$ ratios or converted values. The data were processed using Microsoft Excel software (Microsoft, Bellevue, WA, USA) and the MDI gene-expression analysis software package (MicroDiagnostic).

Hierarchical clustering and construction of the gene expression scoring system. Step 1: Genes with fluorescence intensity values below the detection limit in two or more of the 12 PDAC and 4 IPMC specimens and three or more of the 24 IPMN specimens were excluded. Step 2: The mean of the converted values of the chosen genes were calculated for each group, and the genes that met the following requirement were selected: absolute value of the difference between the mean of 12 PDAC+ four IPMC samples and the mean of 24 IPMN samples $\geq 0.5$. Step 3: $t$-Test was used to compare the converted values between the PDAC plus IPMC samples and the IPMN samples. The genes with a $p<0.01$ were selected. Step 4 : Hierarchical clustering was performed using the Euclidean distance with EpressionView Pro software (MicroDiagnostic). Step 5: For six genes, the mean of the converted values was higher in the IPMN samples than in the other samples, and the converted values were multiplied by -1 . The converted values of the selected genes from all specimens were summed as the gene-expression scores (GSs).

Gene ontology and pathway enrichment analyses. To identify the role of the selected genes from the CGEA, Gene Ontology (GO) and Kyoto Encyclopedia of Genes and Genomes (KEGG) pathway enrichment analyses were performed via the Database for Annotation, Visualization and Integrated Discovery (DAVID 6.8; http://david.abcc.ncifcrf.gov/) (21, 22). GO terms [grouped into the biological process (BP), cellular component (CC) and molecular function (MF) categories] and KEGG pathways with $p<0.05$, which is equivalent to 1.3 of the enrichment score, were considered significantly enriched in these analyses.

Statistical analysis. Continuous variables (i.e. age, tumor size, serum CEA and CA 19-9 levels and GS) are reported as the means \pm standard deviation (SD) and were compared using unpaired Student's $t$-test (two-tailed). Categorical variables (i.e. sex and location of disease) were determined using chi-square statistics. The diagnostic yield of

\section{CGEA 14,400 genes, 40 specimens}

Step 1

Genes below detection limit were excluded

\section{1,614 Genes}

Step 2

Genes with difference of mean converted value $\geq 0.5$ between 2 groups were selected

\section{Genes}

Step 3

\section{Genes with significant difference in mean} converted value between 2 group ( $p$-value $<0.01$ ) were selected

\section{Genes}

Figure 1. Schematic flow chart of gene selection in the comprehensive gene-expression analysis.

CEA and CA 19-9 levels and GS in distinguishing IPMN from pancreatic malignancies (IPMC and PDAC) was assessed using the area under the receiver operating characteristic (ROC) curve (AUC). Additionally, the combined diagnostic yield of GS plus CEA and GS plus CA19-9 was evaluated. A value of $p<0.05$ was considered statistically significant. All statistical analyses were implemented in GraphPad Prism 7.0 (GraphPad, San Diego, CA, USA) and SPSS 26 (IBM, Armonk, NY, USA).

\section{Results}

Diagnostic yield of the gene-expression scoring system. Figure 1 shows the CGEA results of 40 blood specimens; 11,614 genes were selected in step 1, 29 in step 2, and 13 in step 3. The 13 selected genes are described in Table II. Hierarchical clustering analysis clearly categorized two different clusters: 1) the pancreatic malignancy cluster and 2) the IPMN cluster (Figures 2 and 3A). By calculating the GS, it was possible to distinguish pancreatic malignancy specimens from IPMN specimens using this analysis, as shown in Figures 3B and 4. Pancreatic malignancies had high GSs, while most of the IPMNs tended to have lower scores.

ROC analysis showed that the three markers were able to discriminate patients with pancreatic malignancies from those with IPMN, with an AUC of 0.71 for CEA [95\% confidence interval $(\mathrm{CI})=0.53-0.90], 0.93$ for CA 19-9 $(95 \%$ $\mathrm{CI}=0.85-1.00$ ), and 0.94 for $\mathrm{GS}$ (95\% CI=0.86-1.00) (Figure $5 \mathrm{~A})$. In addition, the combined value of CEA plus GS and CA19-9 plus GS showed slight improvement in diagnostic yield, with an AUC of 0.94 for CEA plus GS (95\% CI=0.871.00 ) and 0.97 for CA 19-9 plus GS (95\% CI=0.92-1.00). As shown in Figure 5A, GS showed the highest diagnostic yield compared with the other two tumor markers at the cut-off 
Table II. Thirteen selected genes from the comprehensive gene-expression analysis.

\begin{tabular}{|c|c|c|c|c|}
\hline Expression relative to IPMN & Gene symbol & Gene name & ID & $\begin{array}{l}\text { ENTREZ } \\
\text { GENE ID }\end{array}$ \\
\hline \multirow{7}{*}{$\begin{array}{l}\text { Higher in pancreatic } \\
\text { malignancies }\end{array}$} & DSC2 & Desmocollin 2, transcript variant Dsc2b, mRNA. & NM_004949 & 1824 \\
\hline & $J A K 2$ & Janus kinase 2, mRNA. & NM_004972 & 3717 \\
\hline & ANXA3 & Annexin A3, mRNA. & NM_005139 & 306 \\
\hline & TRIM22 & Tripartite motif-containing 22 , mRNA. & NM_006074 & 10346 \\
\hline & $N R G N$ & $\begin{array}{l}\text { Neurogranin (protein kinase } \mathrm{C} \text { substrate, } \mathrm{RC} 3 \text { ), } \\
\text { transcript variant } 1, \text { mRNA. }\end{array}$ & NM_006176 & 4900 \\
\hline & S100A12 & S100 calcium-binding protein A12, mRNA. & NM_005621 & 6283 \\
\hline & TMEM176B & Transmembrane protein $176 \mathrm{~B}$, transcript variant 1, mRNA. & NM_014020 & 28959 \\
\hline \multirow{6}{*}{$\begin{array}{l}\text { Lower in pancreatic } \\
\text { malignancies }\end{array}$} & CBLN3 & Similar to CBLN3 (LOC341816), mRNA. & XM_292223 & 643866 \\
\hline & $H I P 1 R$ & $\begin{array}{l}\text { cDNA FLJ33218 fis, clone ASTRO2000381, } \\
\text { highly similar to Mus musculus } \\
\text { Huntington-interacting protein 1-related, mRNA. }\end{array}$ & AK090537 & 9026 \\
\hline & PTDSS2 & Phosphatidylserine synthase 2 (PTDSS2), mRNA. & NM_030783 & 81490 \\
\hline & MS4A1 & $\begin{array}{l}\text { Membrane spanning 4-domains, subfamily A, } \\
\text { member } 1 \text {, transcript variant } 3 \text {, mRNA. }\end{array}$ & NM_021950 & 931 \\
\hline & KITLG & cDNA FLJ31341 fis, clone MESAN1000050. & AK055903 & 4254 \\
\hline & $E M C 1$ & KIAA0090, mRNA. & NM_015047 & 23065 \\
\hline
\end{tabular}

IPMN: Intraductal papillary mucinous neoplasm.

value of 14.6 , and the sensitivity and specificity were $94.0 \%$ and $92.0 \%$, respectively. The combinations, especially of CA19-9 plus GS, also slightly improved the diagnostic yield.

Validation of the gene-expression scoring system. ROC curve analysis of the GS using the validation set showed a slightly reduced diagnostic yield at an optimal cut-off score of 11.5, with an AUC of 0.82 (95\% CI=0.58-1.00), a sensitivity of $78.0 \%$, and a specificity of $100 \%$ (Figure 5B). However, the combinations of CEA plus GS and CA19-9 plus GS showed similarly high diagnostic yields, with an AUC of 0.98 for CEA plus GS (95\% CI=0.91-1.00) and 0.93 for CA 19-9 plus GS $(95 \% \mathrm{CI}=0.79-1.00)$.

Detection of early-stage pancreatic malignancies using the gene scoring system. To clarify whether GS was useful in distinguishing IPMN and cStage I-II pancreatic malignancies, we evaluated the diagnostic yield of the three biomarkers, as shown in Figure 6. For this analysis, we enrolled seven patients with cStage I-II pancreatic malignancies and 29 patients with IPMN from both the discovery and validation sets. ROC analysis showed that these three markers were able to discriminate between patients with cancer and controls, with AUCs of 0.65 for CEA (95\% CI=0.35-0.95), 0.79 for CA 19-9 (95\% CI=0.62$0.97)$, and 0.88 (95\% CI=0.73-1.03) for GS. As shown in Figure 4, GS showed the highest diagnostic yield compared with the other two tumor markers at the cut-off value of 14.8 , and the sensitivity and specificity were $85.0 \%$ and $93.0 \%$, respectively.
$G O$ and KEGG pathway enrichment analysis. GO analysis was performed using the DAVID platform. Figure 7 lists the five significantly up-regulated functions in pancreatic malignancies compared to IPMN, divided into the GO BP (GO_BP in Figure 6A) and GO MF (GO_MF in Figure 7A) categories. Figure $7 \mathrm{~B}$ shows the most significantly downregulated function in pancreatic malignancies compared to IPMN (GO_CC). In brief, the significantly up-regulated functions were related to key regulators of the inflammatory response, such as nuclear factor-kappa B (NF-kB) and interferon-gamma (IFN $\gamma$ ). Regarding KEGG pathway analysis, five genes were found to be registered in the KEGG database (Table III). Both KITLG and MS4Al were involved in the 'hematopoietic cell lineage', with a significant enrichment score of $1.6(p=0.025)$.

\section{Discussion}

Accurate blood-based diagnostic biomarkers are ideal methods for cancer screening programs since they can provide objective data for clinicians in a less invasive manner. We applied a novel gene-scoring system using blood specimens to distinguish both IPMC and PDAC from BD-IPMN. The scoring system, utilizing the expression levels of 13 genes, showed a high diagnostic yield ( sensitivity $=94.0 \%$, specificity $=92.0 \%$ and AUC $=0.94$ ), which was confirmed in the validation set. Furthermore, its diagnostic yield was not reduced even in early-stage pancreatic malignancies (sensitivity $=85.0 \%$, specificity 93.0\% and $\mathrm{AUC}=0.88$ ). 

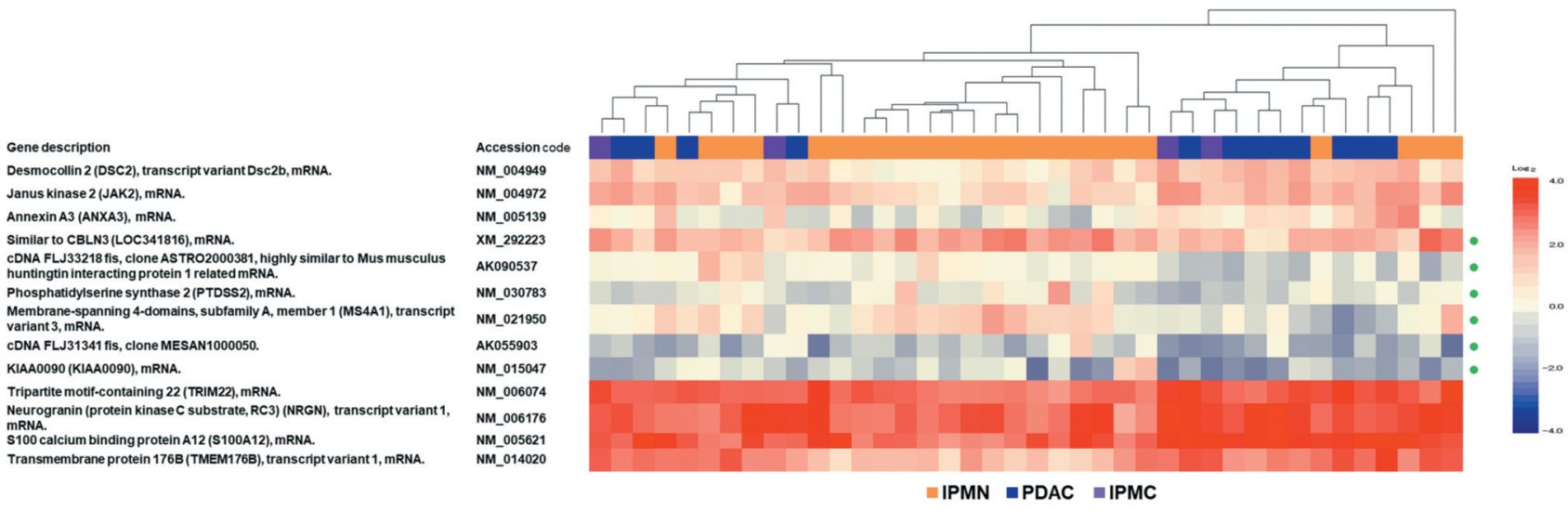

Figure 2. Hierarchical clustering analysis of the discovery set. Hierarchical clustering analysis $(n=40)$ clearly categorized two different clusters: Pancreatic malignancy, and intraductal papillary mucinous neoplasm (IPMN). The color bar on the right side of the figure represents the grades of the relative expression levels: increase (red) and decrease (blue). The means of the converted values for the genes were higher in the IPMN samples than in the IPMNderived carcinoma (IPMC) samples, and pancreatic ductal adenocarcinoma (PDAC) is indicated by green dots. GS: Gene score.

A

IPMN $\quad$ PDAC IIPMC

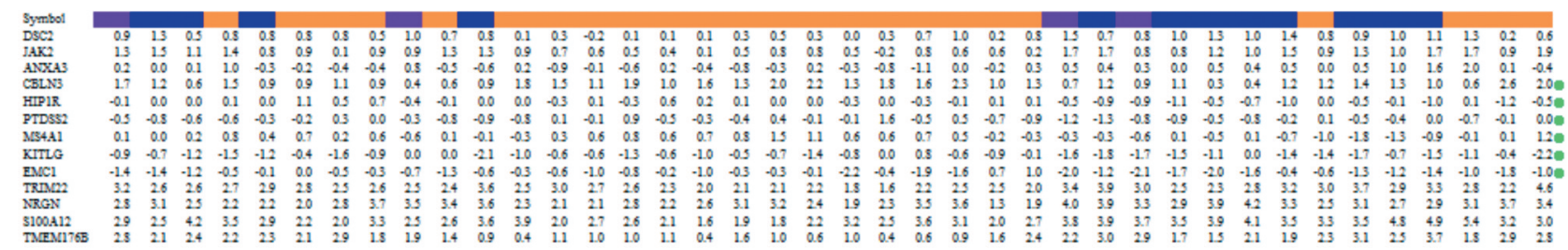

B

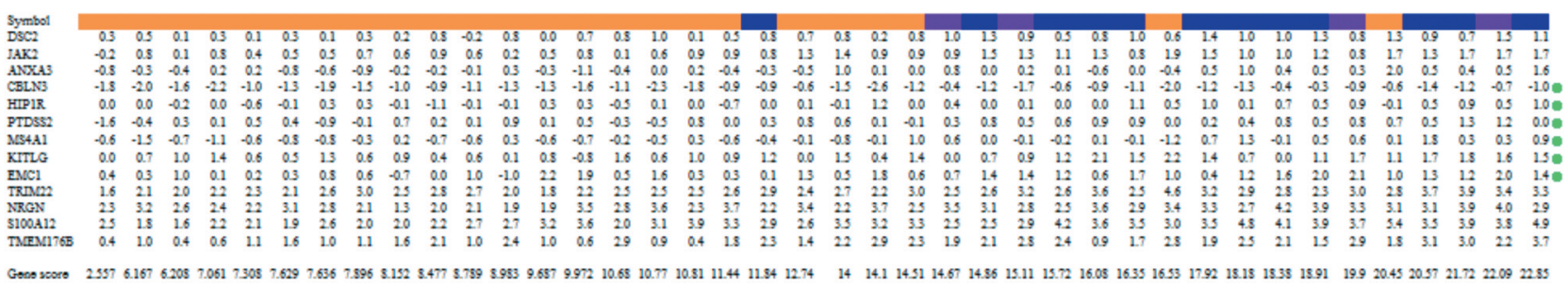

Figure 3. A: Gene-expression ratios converted into $\log _{2}$ values. B: Gene-score calculation. For six genes, the mean of the converted values was higher in intraductal papillary mucinous neoplasm (IPMN) samples than in other samples (green dots), and the converted values were multiplied by -1 . The converted values of the selected genes from all specimens were summed as the gene expression scores. IPMC: IPMN-derived carcinoma; PDAC: pancreatic ductal adenocarcinoma.

Several studies have been conducted aiming to distinguish benign from malignant IPMN. Since bloodbased biomarkers have failed to demonstrate reliable diagnostic yields, recent studies have tended to evaluate samples obtained directly from the pancreatic duct or cystic fluid. According to a systematic review, some biomarkers (e.g. cytology and CEA levels in pancreatic juice or cystic fluid) showed high specificity but the sensitivity was generally low, possibly due to degraded and scant cellular specimens (17). Additionally, endoscopic techniques such as endoscopic ultrasound-guided fineneedle aspiration and ERCP are invasive and may cause life-threatening adverse events such as post-ERCP pancreatitis or tumor cell dissemination. Therefore, blood- 


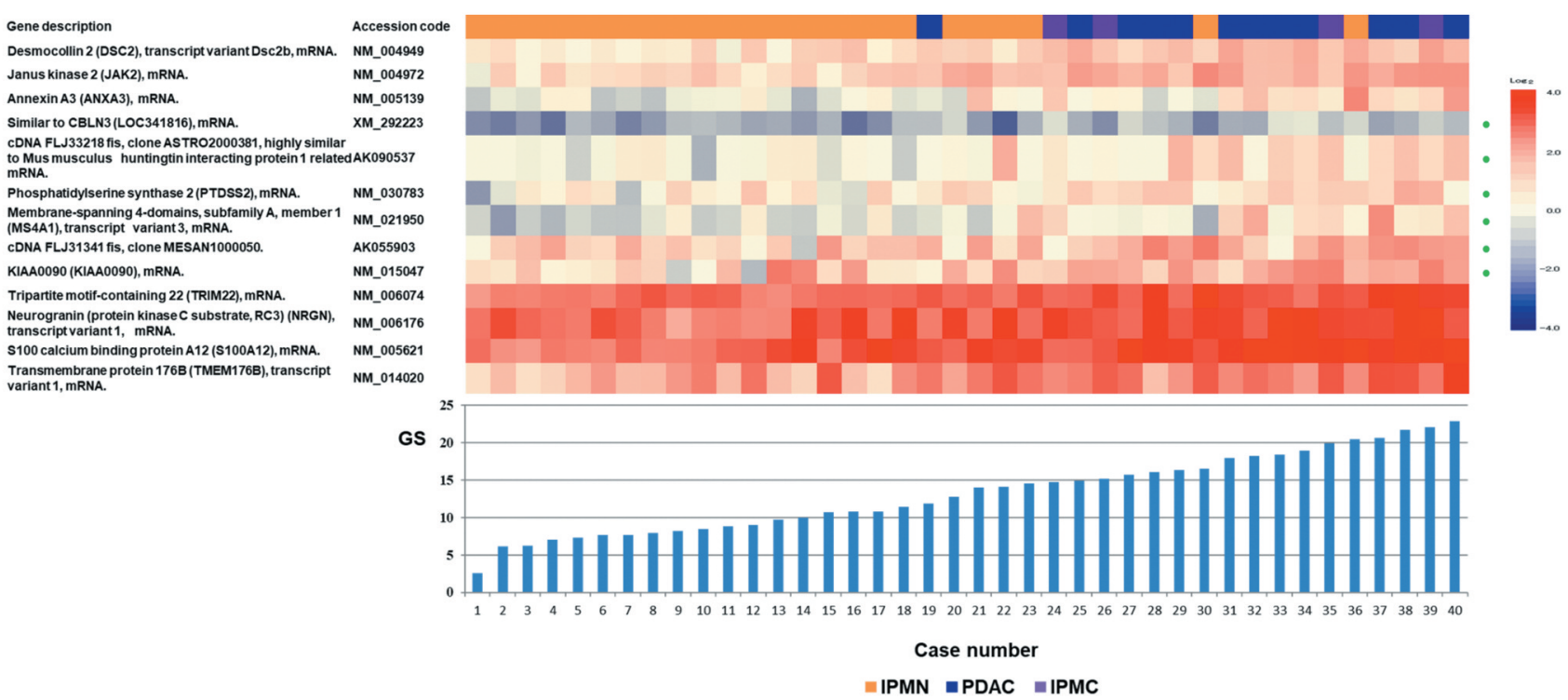

Figure 4. Comprehensive gene-expression analysis of the discovery set $(n=40)$. Specimens and genes are aligned in the order defined by the results of the clustering analysis. The color bar on the right side of the figure represents the grades of the relative expression levels: increase (red) and decrease (blue). The means of the converted values for the genes were higher in the intraductal papillary mucinous neoplasm (IPMN) samples than in the other IPMN samples, as indicated by green dots. IPMC: IPMN-derived carcinoma; PDAC: pancreatic ductal adenocarcinoma; GS: gene score.

based biomarkers have regained attention for the diagnosis of pancreatic neoplasms.

Recent advancements in technology enable us to detect and quantify extremely small amounts of nucleic acids $[e . g$. deoxyribonucleic acid (DNA) and ribonucleic acid (RNA)] or fractions of them in the blood. Regarding RNA, many researchers have evaluated the diagnostic yield of noncoding RNAs, especially microRNAs (miRs), in the blood to establish new biomarkers for IPMN cancer screening. Komatsu et al. reported that plasma miR-223 levels discriminated the malignant potential between benign IPMN and malignant IPMN, as well as the progressive extent of invasiveness between malignant IPMN and PDAC (23). Another group showed that the combination of imaging features with a cluster of miRs might improve the prediction of malignant IPMN (AUC=0.92) (24). Additionally, circulating cell-free DNA is an attractive target for biomarker research. Berger et al. measured the level of circulating cellfree DNA in patients with IPMN and found that it had a high discriminatory power for control versus IPMN as well as for control versus PDAC; however, the diagnostic performance for IPMN versus PDAC was not so high $(75.0 \%$ sensitivity and $71.4 \%$ specificity) (25). Overall, these biomarkers may be promising but require further improvement.

While RNA isolated directly from whole blood may produce increased noise in gene expression and reduced sensitivity compared with total RNA isolated after various protocols, the advantages of profiling gene expression from whole blood are compelling: i) Cell isolation steps that may incidentally alter gene expression patterns are avoided; ii) easy isolation of mRNA from whole blood facilitates studies; and iii) rare subpopulations of cells remain included in the analysis (26). Therefore, we decided to use whole blood for a blood-based screening system that might be faster and more concise than other methods. A similar method was previously utilized to develop a blood-based mRNA screening system for pancreatic cancer (27). In this study, the authors compared the gene expression between PDAC and healthy controls and developed a 56-gene diagnostic system. Even though it showed moderate diagnostic yield for differentiating PDAC and controls (sensitivity $=73.6 \%$ and specificity $=64.7 \%$ ), they found that the assessment of changes in mRNA expression in whole blood was a viable alternative screening strategy for PDAC. Unlike their study, we first focused on IPMN and its related pancreatic malignancies since the screening program for the general population has been considered to be inefficient because of the low lifetime risk of PDAC. Indeed, our newly developed gene-scoring system successfully showed a higher diagnostic yield in distinguishing IPMN and pancreatic malignancies than other biomarkers previously reported.

Regarding the biomolecular background of altered gene expression, GO analysis provided some insights. Among the 13 selected genes in the comprehensive gene expression analysis, seven with higher expression in pancreatic malignancies than IPMN were positively related to five 
A

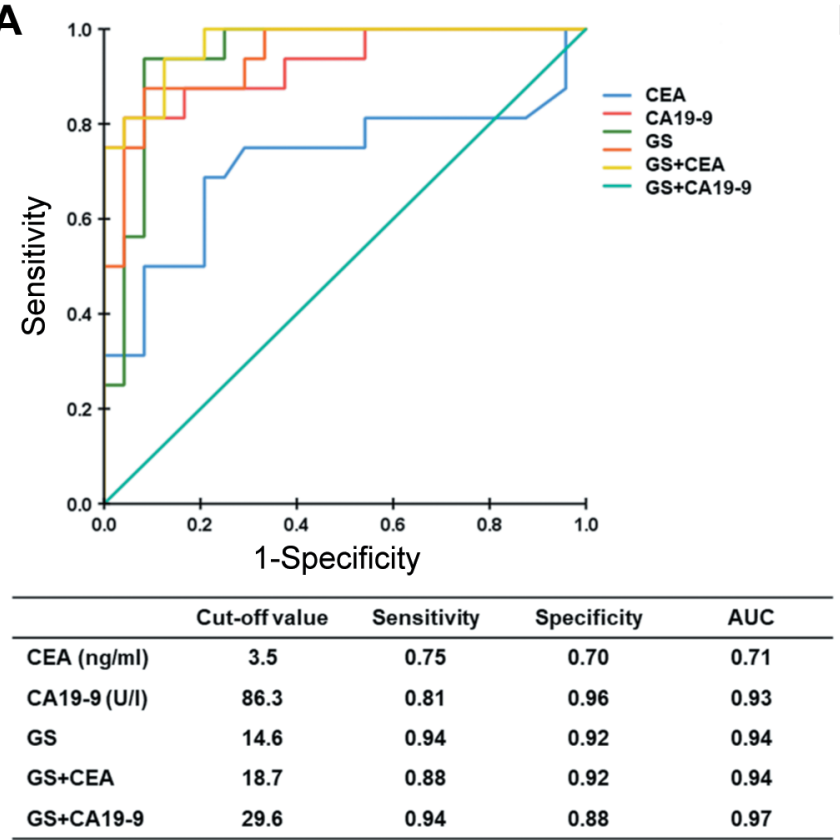

B

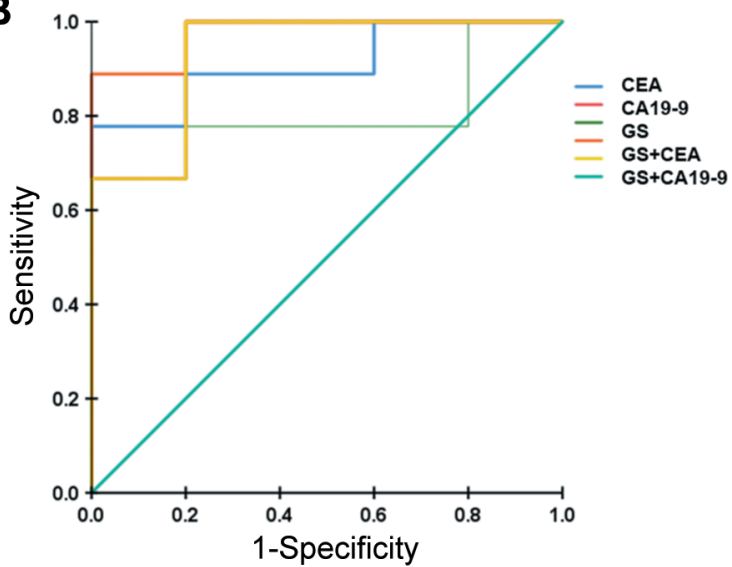

Figure 5. Receiver operating characteristics curve analysis of the discovery and validation sets. A: For the discovery set, the optimal gene score (GS) cut-off was 14.6. The area under the curve (AUC) was 0.94, with a sensitivity of $94.0 \%$ and a specificity of $92.0 \%$. B: For the validation set, the optimal GS cut-off was 8.7. The AUC was 1.00, with a sensitivity of 100\% and a specificity of 100\%. CEA: Carcinoembryonic antigen; CA199: carbohydrate antigen 19-9.

significant functions: NF-kB transcription factor activity, inflammatory response, IFN $\gamma$-mediated signaling pathway, sequence-specific DNA binding transcription factor activity and calcium ion binding. NF- $\mathrm{kB}$ is a transcription factor that has been well studied and is related to carcinogenesis and tumor progression in several cancer types, including PDAC $(28,29)$. In the cytoplasm, NF-kB is inactivated by the inhibitor proteins IkB- $\alpha$ and IkB- $\beta$ and then activated by upstream signals that the IKB inhibitor protein allow to dissociate from NF-kB. These upstream signals can come from the epidermal growth factor receptor tyrosine kinase, interleukin-1 $\beta$ pathway, tumor necrosis factor- $\alpha$ pathway, CD95/tumor necrosis factor-related apoptosis-inducing ligand pathway, or mitogen-activated protein kinases (30). In fact, evidence supports direct and indirect relationships between the malignant progression of IPMN and NF-kB activation (31-33). IFN $\gamma$ is a cytokine whose biological activity via the Janus kinase/signal transducer and activator of transcription (JAK/STAT) pathway is conventionally associated with cytostatic/cytotoxic and antitumor mechanisms during the cell-mediated adaptive immune response (34). As Fukushima et al. reported, the aberrant methylation of the suppressor of cytokine signaling-1 gene, an inhibitor of the JAK/STAT pathway, was found in invasive IPMN but not in benign IPMN, which is suggestive

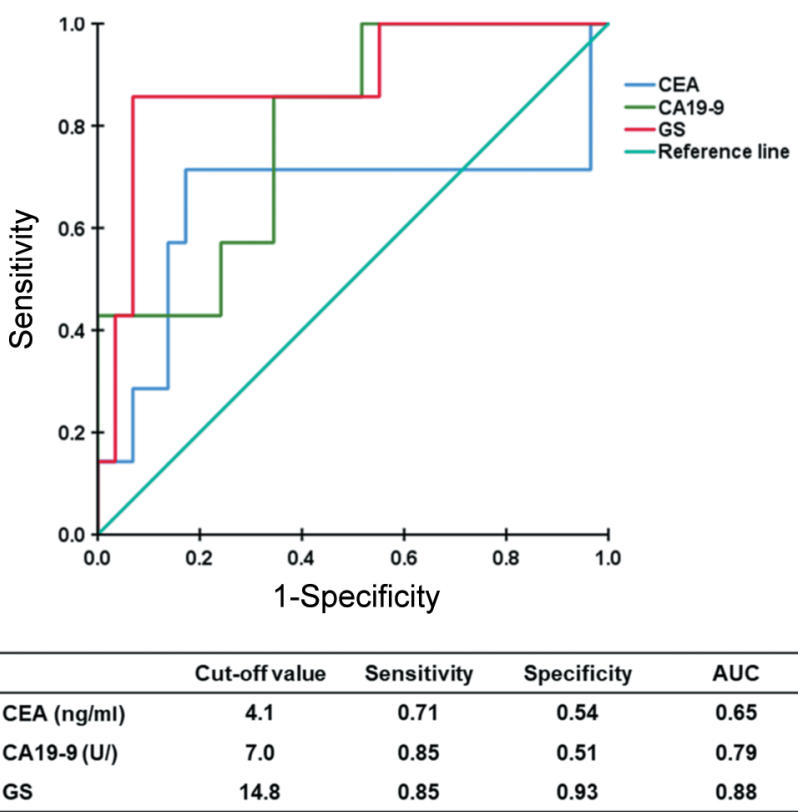

Figure 6. Receiver operating characteristics curve analysis for earlystage pancreatic malignancies. The optimal gene score (GS) cut-off was 14.8. The area under the curve (AUC) was 0.85, with a sensitivity of $93.0 \%$ and a specificity of $88.0 \%$. CEA: Carcinoembryonic antigen; CA19-9: carbohydrate antigen 19-9. 
A

GO BP

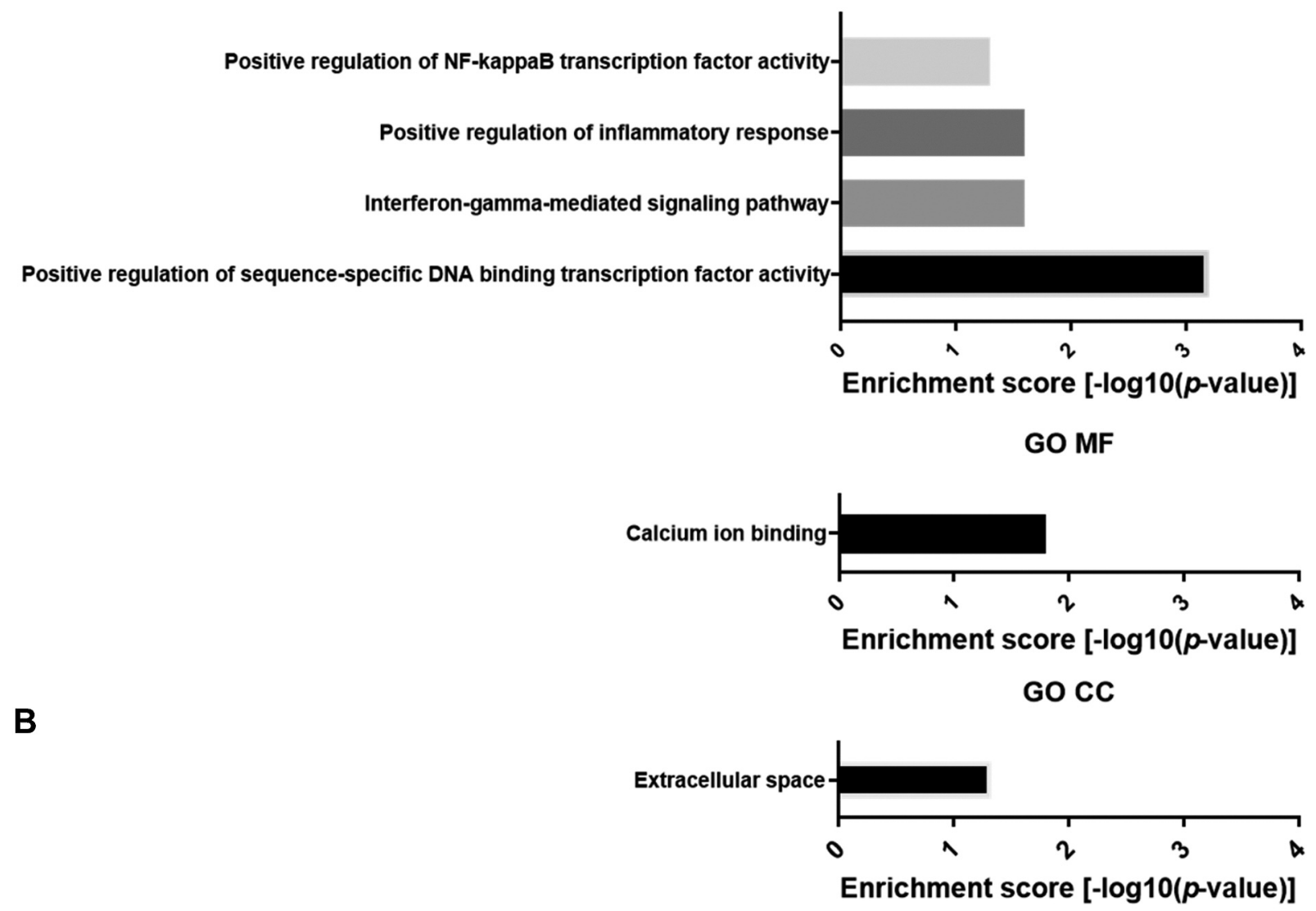

Figure 7. Gene Ontology (GO) analysis. A: Five significantly up-regulated GO functions in pancreatic malignancies compared to intraductal papillary mucinous neoplasm (IPMN) are listed. B: One significantly down-regulated GO function in pancreatic malignancies compared to IPMN is listed. BP: Biological process; MF: molecular function; CC: cellular component.

Table III. Prediction of involved cell signaling pathways.

\begin{tabular}{lc}
\hline Gene name (ENTREZ GENE ID) & KEGG PATHWAY \\
\hline Janus kinase 2 (JAK2) (3717) & $\begin{array}{c}\text { Chemokine signaling pathway, PI3K-AKT signaling pathway, Signaling pathways } \\
\text { regulating the pluripotency of stem cells, JAK-STAT signaling pathway, } \\
\text { Cholinergic synapse, Prolactin signaling pathway, Adipocytokine signaling pathway } \\
\text { RAS signaling pathway, RAP1 signaling pathway, PI3K-AKT signaling pathway, } \\
\text { KIT ligand (KITLG) (4254) }\end{array}$ \\
$\begin{array}{l}\text { Hematopoietic cell lineage, Melanogenesis, Pathways in cancer } \\
\text { Arrhythmogenic right ventricular cardiomyopathy }\end{array}$ \\
$\begin{array}{c}\text { Membrane spanning 4-domains A1 (MS4A1) (931) } \\
\text { Phosphatidylserine synthase 2 (PTDSS2) (81490) }\end{array}$ \\
\hline
\end{tabular}

KEGG: Kyoto Encyclopedia of Genes and Genomes; PI3K: phosphatidylinositol-3 kinase; STAT: signal transducer and activator of transcription; RAP: RAS-proximate-1.

of a relation between the malignant progression of IPMN and IFN $\gamma$. Finally, calcium ions may be uniquely related to IPMN progression via the $\mathrm{S} 100$ protein family, a multigene calcium-binding family (35). S100 protein is related to various pathways, such as mitogen-activated protein kinases, phosphatidylinositol 3-kinase, Rho GTPases, NF-kB and JAK/STAT. Among various S100 proteins, S100P is reported to be highly expressed in IPMN but not in normal pancreatic 
ductal epithelium. Moreover, S100P was clearly expressed in the invasive components of IPMNs, including perineural, lymphatic and minimal invasion. In summary, our genescoring system comprehensively includes genes that have been suggested to be related to IPMN and its progression.

This study has several limitations. Firstly, this was a singlecenter, observational study with a small sample size. Secondly, most of the patients did not undergo surgical resection, and the final diagnosis was not confirmed. Considering the high accuracy of computed tomography and magnetic resonance imaging in distinguishing IPMN from other cyst types, we considered that it was possible to diagnose IPMN by imaging modalities. Regarding patients with IPMC, four out of seven patients underwent surgical resection, and pathological diagnosis was confirmed. The other three patients had a distinct intramural mass of more than $10 \mathrm{~mm}$ with infiltration to the pancreatic parenchyma or celiac artery, which was highly suggestive of the malignant transformation of BD-IPMN. Thirdly, the GS was measured once in all patients, and changes in the GS along with the malignant transformation of BDIPMN during surveillance were not evaluated. Further large studies are warranted to confirm the results.

In conclusion, we developed a novel blood-based geneexpression scoring system based on various pro-tumoral molecular activities. To clarify whether this test may be an alternative for conventional tumor markers or imaging modalities in the cancer screening of patients with IPMN, further prospective studies should be undertaken in the nearest future.

\section{Conflicts of Interest}

The Nippon Gene Co., Ltd. provided support in the form of salary for one of the Authors (RH) but did not have any additional roles in the current study. Other Authors have nothing to declare for this study.

\section{Authors' Contributions}

RS, HT, JI and SW conceived the presented idea. RS, HT, RH, JI and SW developed the theory and performed the computations. RS, HT, JI and SW verified the analytical methods. RS, NK, HI, TT, MS, HA, YS, YO, JN, MT, TK, MH, TH provided medical care to patients. HO supervised the findings of this work. All Authors discussed the results and contributed to the final article.

\section{Acknowledgements}

The Authors greatly appreciate Ms. Chikako Sato and Ms. Rie Hikichi for their skillful assistance in the study.

\section{References}

1 Tanaka M: International consensus guidelines for the management of IPMN and MCN of the pancreas. Nihon Shokakibyo Gakkai Zasshi 104(9): 1338-1343, 2007. PMID: 17827904.
2 Tanaka M, Fernandez-Del Castillo C, Kamisawa T, Jang JY, Levy P, Ohtsuka T, Salvia R, Shimizu Y, Tada M and Wolfgang CL: Revisions of International Consensus Fukuoka Guidelines for the management of IPMN of the pancreas. Pancreatology 17(5): 738753, 2017. PMID: 28735806. DOI: 10.1016/j.pan.2017.07.007

3 Oyama H, Tada M, Takagi K, Tateishi K, Hamada T, Nakai Y, Hakuta R, Ijichi H, Ishigaki K, Kanai S, Kogure H, Mizuno S, Saito K, Saito T, Sato T, Suzuki T, Takahara N, Morishita Y, Arita J, Hasegawa K, Tanaka M, Fukayama M and Koike K: Long-term risk of malignancy in branch-duct intraductal papillary mucinous neoplasms. Gastroenterology 158(1): 226-237 e225, 2020. PMID: 31473224. DOI: $10.1053 /$ j.gastro.2019.08.032

4 Vege SS, Ziring B, Jain R, Moayyedi P, Clinical Guidelines C and American Gastroenterology A: American Gastroenterological Association institute guideline on the diagnosis and management of asymptomatic neoplastic pancreatic cysts. Gastroenterology 148(4): 819-822, 2015. PMID: 25805375. DOI: 10.1053/j.gastro. 2015.01 .015

5 Del Chiaro M, Verbeke C, Salvia R, Kloppel G, Werner J, McKay C, Friess H, Manfredi R, Van Cutsem E, Lohr M, Segersvard R and European Study Group on Cystic Tumours of the P: European Experts Consensus Statement on cystic tumours of the pancreas. Dig Liver Dis 45(9): 703-711, 2013. PMID: 23415799. DOI: 10.1016/j.dld.2013.01.010

6 Yamada S, Fujii T, Murotani K, Kanda M, Sugimoto H, Nakayama G, Koike M, Fujiwara M, Nakao A and Kodera Y: Comparison of the international consensus guidelines for predicting malignancy in intraductal papillary mucinous neoplasms. Surgery 159(3): 878-884, 2016. PMID: 26506564. DOI: $10.1016 /$ j.surg.2015.08.042

7 Xu MM, Yin S, Siddiqui AA, Salem RR, Schrope B, Sethi A, Poneros JM, Gress FG, Genkinger JM, Do C, Brooks CA, Chabot JA, Kluger MD, Kowalski T, Loren DE, Aslanian H, Farrell JJ and Gonda TA: Comparison of the diagnostic accuracy of three current guidelines for the evaluation of asymptomatic pancreatic cystic neoplasms. Medicine 96(35): e7900, 2017. PMID: 28858107. DOI: 10.1097/MD .0000000000007900

8 Ma GK, Goldberg DS, Thiruvengadam N, Chandrasekhara V, Kochman ML, Ginsberg GG, Vollmer CM and Ahmad NA: Comparing American Gastroenterological Association pancreatic cyst management guidelines with Fukuoka Consensus Guidelines as predictors of advanced neoplasia in patients with suspected pancreatic cystic neoplasms. J Am Coll Surg 223(5): 729-737 e721, 2016. PMID: 27497827. DOI: 10.1016/j.jamcollsurg.2016.07.011

9 Singhi AD, Zeh HJ, Brand RE, Nikiforova MN, Chennat JS, Fasanella KE, Khalid A, Papachristou GI, Slivka A, Hogg M, Lee KK, Tsung A, Zureikat AH and McGrath K: American Gastroenterological Association guidelines are inaccurate in detecting pancreatic cysts with advanced neoplasia: A clinicopathologic study of 225 patients with supporting molecular data. Gastrointest Endosc 83(6): 1107-1117 e1102, 2016. PMID: 26709110. DOI: 10.1016/j.gie.2015.12.009

10 Ge PS, Muthusamy VR, Gaddam S, Jaiyeola DM, Kim S, Sedarat A, Donahue TR, Hosford L, Wilson RH, Grande DP, Keswani RN, Kushnir VM, Mullady D, Edmundowicz SA, Early DS, Komanduri S, Wani S and Watson RR: Evaluation of the 2015 AGA guidelines on pancreatic cystic neoplasms in a large surgically confirmed multicenter cohort. Endosc Int Open 5(3): E201-E208, 2017. PMID: 28317015. DOI: 10.1055/s-0042122010 
11 Takano S, Fukasawa M, Maekawa S, Kadokura M, Miura M, Shindo H, Takahashi E, Sato $\mathrm{T}$ and Enomoto N: Deep sequencing of cancer-related genes revealed GNAS mutations to be associated with intraductal papillary mucinous neoplasms and its main pancreatic duct dilation. PLoS One 9(6): e98718, 2014. PMID: 24897499. DOI: 10.1371/journal.pone.0098718

12 Hayakawa H, Fukasawa M, Sato T, Takano S, Kadokura M, Shindo H, Takahashi E, Hirose S, Kawakami S, Fukasawa Y, Maekawa S, Inoue T, Yamaguchi T, Nakayama Y, Kawaida H, Kono H, Mochizuki K, Kondo T, Ichikawa D and Enomoto N: Carcinoembryonic antigen level in the pancreatic juice is effective in malignancy diagnosis and prediction of future malignant transformation of intraductal papillary mucinous neoplasm of the pancreas. J Gastroenterol 54(11): 1029-1037, 2019. PMID: 31111221. DOI: 10.1007/s00535-019-01592-8

13 Al-Haddad M, DeWitt J, Sherman S, Schmidt CM, LeBlanc JK, McHenry L, Cote G, El Chafic AH, Luz L, Stuart JS, Johnson CS, Klochan C and Imperiale TF: Performance characteristics of molecular (DNA) analysis for the diagnosis of mucinous pancreatic cysts. Gastrointest Endosc 79(1): 79-87, 2014. PMID: 23845445. DOI: 10.1016/j.gie.2013.05.026

14 Hata T, Dal Molin M, Hong SM, Tamura K, Suenaga M, Yu J, Sedogawa H, Weiss MJ, Wolfgang CL, Lennon AM, Hruban RH and Goggins MG: Predicting the grade of dysplasia of pancreatic cystic neoplasms using cyst fluid DNA methylation markers. Clin Cancer Res 23(14): 3935-3944, 2017. PMID: 28148542. DOI: $10.1158 / 1078-0432 . C C R-16-2244$

15 Maire F, Voitot H, Aubert A, Palazzo L, O'Toole D, Couvelard A, Levy P, Vidaud M, Sauvanet A, Ruszniewski P and Hammel P: Intraductal papillary mucinous neoplasms of the pancreas: Performance of pancreatic fluid analysis for positive diagnosis and the prediction of malignancy. Am J Gastroenterol 103(11): 2871-2877, 2008. PMID: 18775021. DOI: 10.1111/j.15720241.2008.02114.x

16 Carrara S, Cangi MG, Arcidiacono PG, Perri F, Petrone MC, Mezzi G, Boemo C, Talarico A, Cin ED, Grassini G, Doglioni $\mathrm{C}$ and Testoni PA: Mucin expression pattern in pancreatic diseases: Findings from EUS-guided fine-needle aspiration biopsies. Am J Gastroenterol 106(7): 1359-1363, 2011. PMID: 21647207. DOI: 10.1038/ajg.2011.22

17 Tanaka M, Heckler M, Liu B, Heger U, Hackert T and Michalski $\mathrm{CW}$ : Cytologic analysis of pancreatic juice increases specificity of detection of malignant IPMN-a systematic review. Clin Gastroenterol Hepatol 17(11): 2199-2211 e2121, 2019. PMID: 30630102. DOI: 10.1016/j.cgh.2018.12.034

18 Tada T, Honma R, Imai JI, Saze Z, Kogure M, Marubashi S, Tasaki K, Unakami M, Ezaki J, Tamura H, Nishikawa A, Hashimoto Y, Waguri S, Watanabe S and Gotoh M: A novel gene expression scoring system for accurate diagnosis of basaloid squamous cell carcinoma of the esophagus. Int $\mathbf{J}$ Oncol 51(3): 877-886, 2017. PMID: 28731134. DOI: 10.3892/ ijo.2017.4075

19 Chun YS, Pawlik TM and Vauthey JN: Eighth edition of the AJCC Cancer Staging Manual: Pancreas and hepatobiliary cancers. Ann Surg Oncol 25(4): 845-847, 2018. PMID: 28752469. DOI: $10.1245 / \mathrm{s} 10434-017-6025-\mathrm{x}$

20 Kamarajah SK, Burns WR, Frankel TL, Cho CS and Nathan H: Validation of the American Joint Commission on Cancer (AJCC) eighth edition staging system for patients with pancreatic adenocarcinoma: A surveillance, epidemiology and end results
(SEER) analysis. Ann Surg Oncol 24(7): 2023-2030, 2017. PMID: 28213792. DOI: 10.1245/s10434-017-5810-x

21 Huang da W, Sherman BT and Lempicki RA: Bioinformatics enrichment tools: Paths toward the comprehensive functional analysis of large gene lists. Nucleic Acids Res 37(1): 1-13, 2009. PMID: 19033363. DOI: 10.1093/nar/gkn923

22 Huang da W, Sherman BT, Zheng X, Yang J, Imamichi T, Stephens R and Lempicki RA: Extracting biological meaning from large gene lists with DAVID. Curr Protoc Bioinformatics Chapter 13: Unit 13A.7, 2009. PMID: 22294390. DOI: 10.1002/0471250953.bi1311s27

23 Komatsu S, Ichikawa D, Miyamae M, Kawaguchi T, Morimura R, Hirajima S, Okajima W, Ohashi T, Imamura T, Konishi H, Shiozaki A, Ikoma H, Okamoto K, Taniguchi H and Otsuji E: Malignant potential in pancreatic neoplasm; new insights provided by circulating mir-223 in plasma. Expert Opin Biol Ther 15(6): 773-785, 2015. PMID: 25819175. DOI: $10.1517 / 14712598.2015 .1029914$

24 Permuth JB, Choi J, Balarunathan Y, Kim J, Chen DT, Chen L, Orcutt S, Doepker MP, Gage K, Zhang G, Latifi K, Hoffe S, Jiang K, Coppola D, Centeno BA, Magliocco A, Li Q, Trevino J, Merchant N, Gillies R, Malafa M and Florida Pancreas C: Combining radiomic features with a miRNA classifier may improve prediction of malignant pathology for pancreatic intraductal papillary mucinous neoplasms. Oncotarget 7(52): 85785-85797, 2016. PMID: 27589689. DOI: 10.18632/ oncotarget. 11768

25 Berger AW, Schwerdel D, Costa IG, Hackert T, Strobel O, Lam S, Barth TF, Schroppel B, Meining A, Buchler MW, Zenke M, Hermann PC, Seufferlein T and Kleger A: Detection of hot-spot mutations in circulating cell-free DNA from patients with intraductal papillary mucinous neoplasms of the pancreas. Gastroenterology 151(2): 267-270, 2016. PMID: 27343369. DOI: 10.1053/j.gastro.2016.04.034

26 Vartanian K, Slottke R, Johnstone T, Casale A, Planck SR, Choi D, Smith JR, Rosenbaum JT and Harrington CA: Gene expression profiling of whole blood: Comparison of target preparation methods for accurate and reproducible microarray analysis. BMC Genomics 10: 2, 2009. PMID: 19123946. DOI: 10.1186/1471-2164-10-2

27 Sakai Y, Honda M, Matsui S, Komori O, Murayama T, Fujiwara T, Mizuno M, Imai Y, Yoshimura K, Nasti A, Wada T, Iida N, Kitahara M, Horii R, Toshikatsu T, Nishikawa M, Okafuji H, Mizukoshi E, Yamashita T, Yamashita T, Arai K, Kitamura K, Kawaguchi K, Takatori H, Shimakami T, Terashima T, Hayashi T, Nio K, Kaneko S and Hokuriku Liver Study G: Development of novel diagnostic system for pancreatic cancer, including early stages, measuring mRNA of whole blood cells. Cancer Sci 110(4): 1364-1388, 2019. PMID: 30742728. DOI: 10.1111/cas. 13971

28 Holcomb B, Yip-Schneider M and Schmidt CM: The role of nuclear factor kappab in pancreatic cancer and the clinical applications of targeted therapy. Pancreas 36(3): 225-235, 2008. PMID: 18362834. DOI: 10.1097/MPA.0b013e31815b3207

29 Ling J, Kang Y, Zhao R, Xia Q, Lee DF, Chang Z, Li J, Peng B, Fleming JB, Wang H, Liu J, Lemischka IR, Hung MC and Chiao PJ: KRASG12D-induced IKK2/beta/NF-kappaB activation by IL1alpha and p62 feedforward loops is required for development of pancreatic ductal adenocarcinoma. Cancer Cell 21(1): 105-120, 2012. PMID: 22264792. DOI: 10.1016/j.ccr.2011.12.006 
30 Hayden MS and Ghosh S: Shared principles in NF-kappaB signaling. Cell 132(3): 344-362, 2008. PMID: 18267068. DOI: 10.1016/j.cell.2008.01.020

31 Schonleben F, Qiu W, Bruckman KC, Ciau NT, Li X, Lauerman $\mathrm{MH}$, Frucht H, Chabot JA, Allendorf JD, Remotti HE and Su GH: BRAF and KRAS gene mutations in intraductal papillary mucinous neoplasm/carcinoma (IPMN/IPMC) of the pancreas. Cancer Lett 249(2): 242-248, 2007. PMID: 17097223. DOI: 10.1016/j.canlet.2006.09.007

32 Furukawa T, Kuboki Y, Tanji E, Yoshida S, Hatori T, Yamamoto M, Shibata N, Shimizu K, Kamatani N and Shiratori K: Wholeexome sequencing uncovers frequent gnas mutations in intraductal papillary mucinous neoplasms of the pancreas. Sci Rep 1: 161, 2011. PMID: 22355676. DOI: 10.1038/srep00161

33 Maker AV, Katabi N, Qin LX, Klimstra DS, Schattner M, Brennan MF, Jarnagin WR and Allen PJ: Cyst fluid interleukin- 1beta (IL1beta) levels predict the risk of carcinoma in intraductal papillary mucinous neoplasms of the pancreas. Clin Cancer Res 17(6): 1502-1508, 2011. PMID: 21266527. DOI: 10.1158/10780432.CCR-10-1561

34 Zaidi MR and Merlino G: The two faces of interferon-gamma in cancer. Clin Cancer Res 17(19): 6118-6124, 2011. PMID: 21705455. DOI: 10.1158/1078-0432.CCR-11-0482

35 Ji YF, Huang H, Jiang F, Ni RZ and Xiao MB: S100 family signaling network and related proteins in pancreatic cancer (review). Int J Mol Med 33(4): 769-776, 2014. PMID: 24481067. DOI: $10.3892 /$ ijmm.2014.1633

Received September 10, 2020

Revised September 18, 2020

Accepted September 21, 2020 